\title{
Metamagnetic phase transition of the antiferromagnetic Heisenberg icosahedron
}

\author{
Christian Schrödel* \\ Department of Electrical Engineering and Computer Science, \\ University of Applied Sciences Bielefeld, D-33602 Bielefeld, \\ Germany \& Ames Laboratory, Ames, Iowa 50011, USA \\ Heinz-Jürgen Schmidt and Jürgen Schnack \\ Universität Osnabrück, Fachbereich Physik, D-49069 Osnabrück, Germany \\ Marshall Luban \\ Ames Laboratory 85 Department of Physics and Astronomy, Iowa State University, Ames, Iowa 50011, USA
}

(Dated: October 3, 2018)

\begin{abstract}
The observation of hysteresis effects in single molecule magnets like $\mathrm{Mn}_{12}$-acetate has initiated ideas of future applications in storage technology. The appearance of a hysteresis loop in such compounds is an outcome of their magnetic anisotropy. In this Letter we report that magnetic hysteresis occurs in a spin system without any anisotropy, specifically, where spins mounted on the vertices of an icosahedron are coupled by antiferromagnetic isotropic nearest-neighbor Heisenberg interaction giving rise to geometric frustration. At $T=0$ this system undergoes a first order metamagnetic phase transition at a critical field $B_{c}$ between two distinct families of ground state configurations. The metastable phase of the system is characterized by a temperature and field dependent survival probability distribution.
\end{abstract}

PACS numbers: 75.50.Xx;75.10.Hk;75.10.Jm;75.30.Cr

Keywords: Heisenberg model, Metamagnetic Phase Transition

Introduction-Low-dimensional magnetic systems show a variety of fascinating phenomena that are associated with geometrical frustration 1, 2]. Among them are magnetization plateaus and jumps as well as unusual susceptibility minima, as observed for example for the kagome lattice antiferromagnet [3, 4]. Some of these effects can also occur in certain strongly frustrated magnetic molecules such as the Keplerate $\left\{\mathrm{Mo}_{72} \mathrm{Fe}_{30}\right\}[5]$. In this Letter we report that a first order metamagnetic phase transition (with associated hysteresis and metastability effects) occurs for a system of spins that are mounted on the vertices of an icosahedron when an external magnetic field, $B$, equals a critical value $B_{\text {c. }}$ These spins interact with one another only via nearest-neighbor, antiferromagnetic isotropic Heisenberg exchange, but due to the geometrical frustration originating in the unique geometry of the icosahedron they undergo the metamagnetic transition despite the absence of any anisotropic energy terms. As the field proceeds through a closed cycle the magnetization traces the hysteresis loop shown in Fig. 11 The metastable phases, including the temperature dependence of their lifetime, show rich characteristics.

Our exact classical treatment shows that the abrupt transition at $T=0$ originates in the intersection of two energy curves belonging to different families of spin configurations that are ground states below and above the critical field. The minimum of the two energy functions constitutes a non-convex minimal energy function of the spin system and this gives rise to a metamagnetic phase transition $[6]$. At $T=0$ the partition function is a non-

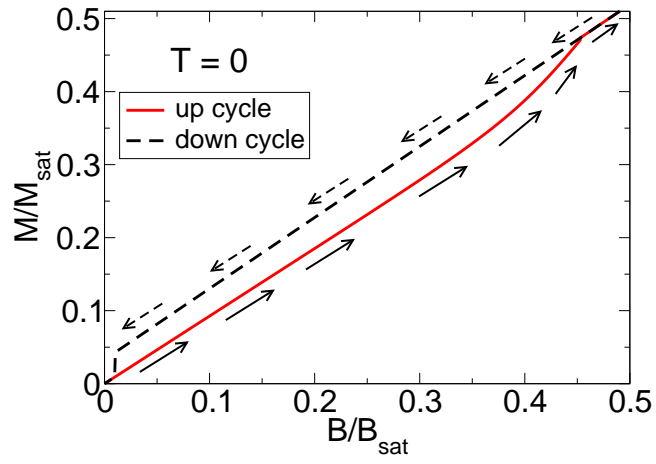

FIG. 1: Hysteresis behavior of the classical icosahedron in an applied magnetic field obtained by classical spin dynamics simulations.

analytic function of $B$, and since the magnetization features a finite jump at $B_{\mathrm{c}}$ the transition is of first order. We also show that the corresponding quantum spin system for sufficiently large spin quantum number $s$ possesses a non-convex set of lowest energy levels when plotted versus total spin. This is the discrete analog of the non-convex classical minimal energy function. Therefore, also the quantum spin system features an unusual magnetization jump.

Classical simulations at $T=0$ - The behavior of classical spin systems subject to an applied magnetic field both at $T \approx 0$ and finite temperatures can be very effectively studied with the help of a stochastic spin dynamics approach such as that proposed in [7]. Here, the spin system is coupled to a heat bath in a Langevin-type ap- 
proach by using a Landau-Lifshitz-damping term as well as a fluctuating force with white noise characteristics. Starting from an arbitrary initial configuration the spin system can be investigated either at zero temperature by observing the relaxation to its ground state or at finite temperature by following its time evolution.

We consider first $T=0$. For $B=0$ we find, that the spins adopt a non-coplanar configuration, where each of the 12 spin vectors makes an angle of $\arccos (-1 / \sqrt{5}) \approx$ $116.6^{\circ}$ with respect to its five nearest neighbors. This is in agreement with the analytical results derived in [8].

The spins are also subjected to an external magnetic field that increases from zero linearly with time but at a very slow rate. The dynamical evolution of the spins is monitored as a function of time and the results are stored within an animation file [15] enabling direct visualization of the spin vectors. In the first stage the configuration of the spin vectors evolves continuously with $B$ until suddenly there is an abrupt change in their orientations. In the new configuration two spin vectors at opposite ends of a diameter of the icosahedron are aligned parallel to the field while all of the remaining 10 spin vectors subtend a common polar angle with respect to $\vec{B}$ and their azimuthal angles are uniformly spaced. With increasing $B$ the common polar angle decreases monotonically while the azimuthal angles remain fixed until at saturation all spin vectors are parallel to $\vec{B}$.

Analytical results - For a classical spin system the ground states are defined as states $\vec{s}=(\vec{s}(1), \ldots, \vec{s}(N))$ which minimize the energy

$$
\begin{aligned}
H(\vec{s}, B) & =H_{0}(\vec{s})-B \sum_{\mu} s_{z}(\mu) \\
& =\sum_{\mu, \nu} J_{\mu \nu} \vec{s}(\mu) \cdot \vec{s}(\nu)-B \sum_{\mu} s_{z}(\mu) .
\end{aligned}
$$

The coupling $J_{\mu \nu}$ between spins $\mu, \nu$ is chosen as $J=1$ between nearest-neighbors and zero otherwise. Accordingly the magnetic field is given in appropriate units.

The (degenerate) ground states must fulfill the following necessary condition, compare Eq. (21) in [8],

$$
\sum_{\nu} J_{\mu \nu} \vec{s}(\nu)=\kappa_{\mu} \vec{s}(\mu)+\frac{1}{2} \vec{B} \quad(\mu=1, \ldots, N),
$$

where $\kappa_{\mu}$ denote suitable Lagrange parameters. Although this system of equations can only be solved numerically in most cases, we are confident that the following statements about ground states of the icosahedron subject to magnetic fields are correct [9].

As remarked above, the spin configuration for $B=0$ has been established $[8]$. The orientation of the individual spins is such that they form four groups of three spins where each group $i$ is characterized by a common polar angle $\theta_{i}$ and uniformly spaced azimuthal angles.

For $B>0$ we numerically solve (2) with the assumption that the azimuthal angles remain fixed and only the four polar angles vary [16]. Thus we obtain a 1-parameter family (the " $4-\theta$-family") of possible ground states. Interestingly, it provides a local minimum of the energy only for $0 \leq M \leq 5.61441$. One might assume that the $4-\theta$ family also provides a global minimum of energy, i.e. a ground state, for the same interval $0 \leq M \leq 5.61441$, but this is wrong: There exists a different 1-parameter family of solutions of (2) which has a lower energy than the 4- $\theta$-family for $M>M_{0} \simeq 4.92949$. As remarked above, this family can be characterized by two spin vectors which are aligned parallel to $\vec{B}$ and 10 spin vectors with a common polar angle $\theta$ and uniformly spaced relative azimuthal angles. The end points of the latter 10 spin vectors form a regular decagon, and we will call this set of states the "decagon family". As discussed below, the decagon family provides a local minimum of the energy for $0.54102 \leq M \leq 12$. These families give rise to two convex curves in the $E$ vs. $M$ diagram, $E_{1}(M)$ for the 4- $\theta$-family and $E_{2}(M)$ for the decagon-family, which intersect at the point with coordinates $\left(M_{0}, E_{0}\right)$, where $M_{0} \simeq 4.92949$ and $E_{0} \simeq-11.150$.

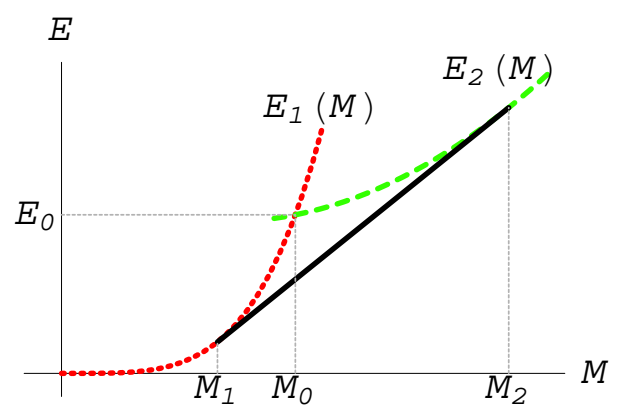

FIG. 2: Schematic representation of two minimal energy curves $E_{1}(M)$ and $E_{2}(M)$, whose minimum $E_{\min }(M)$ is not convex. Their common tangent (solid line) has a slope of $B_{\mathrm{c}}$.

We have strong numerical evidence that the minimum of the two curves provides the absolute minimum $E_{\min }(M)$ of $H_{0}(\vec{s})$ for given $M[9]$. The latter function $E_{\min }(M)$ is therefore not convex and this translates to a jump in the magnetization, $\Delta M=M_{2}-M_{1}$, at a critical field $B_{\mathrm{c}}$, compare [6]. One can identify $B_{\mathrm{c}}$ as the slope of the common tangent of the curves $E_{1}(M)$ and $E_{2}(M)$ illustrated in Fig. 2] This construction is fully equivalent to the statement that the total energies of the two phases, $E_{1}\left(M_{1}\right)-B M_{1}$ and $E_{2}\left(M_{2}\right)-B M_{2}$, are equal for $B=B_{\mathrm{c}}$. According to the Ehrenfest classification the phase transition is of first order. Equivalently, $B_{\mathrm{c}}$ can be obtained by a Maxwell construction in the $M$ vs. $B$ diagram. The pertinent quantities for the phase transition are given in Table 【

Since $M(B)$ has a jump at $B=B_{\mathrm{c}}$ the susceptibility $\chi=\frac{d M}{d B}$ diverges at $B=B_{\mathrm{c}}$ and $T=0$. It is rather easy to derive the leading temperature dependence of this divergence for $T \rightarrow 0$. Recall that an alternative expression 
TABLE I: Characteristic values of the first-order phase transition of the spin icosahedron, compare Fig. $2 B_{\text {sat }}=2(5+\sqrt{5})$ denotes the saturation field. $\chi_{i}=\left.\frac{d M_{i}}{d B}\right|_{B=B_{\mathrm{c}}}, i=1,2$ denote the limit values of the susceptibility.

$$
\begin{array}{ll}
B_{\mathrm{c}} \simeq 5.87614 & B_{\mathrm{c}} \simeq 0.40603 B_{\mathrm{sat}} \\
M_{1} \simeq 4.71461 & M_{2} \simeq 5.10784 \\
E_{1}\left(M_{1}\right) \simeq-12.4324 & E_{2}\left(M_{2}\right) \simeq-10.1218 \\
E_{0} \simeq-11.150 & M_{0} \simeq 4.92949 \\
\chi_{1} \simeq 1.13294 & \chi_{2}=\frac{5}{4+\sqrt{5}} \simeq 0.8018
\end{array}
$$

for the susceptibility is

$$
\chi(B, \beta)=\beta\left(\left\langle M^{2}\right\rangle_{\rho}-\langle M\rangle_{\rho}^{2}\right) \equiv \beta \sigma^{2}(M),
$$

where \langle\rangle$_{\rho}$ denotes the thermal expectation value w. r. t. the canonical ensemble $\rho=\rho(\beta, B)$. In the case of a metamagnetic phase transition of the kind described above the ground state is degenerate for $T \rightarrow 0$ and $B \rightarrow B_{\mathrm{c}}$. Hence $\sigma^{2}(M)$ remains finite in this limit and $\chi\left(B_{\mathrm{c}}, \beta\right)$ diverges linearly with $\beta$, i.e. the critical exponent is 1 .

Stability and hysteresis - In order to investigate the stability of the two families of ground states we performed a standard stability analysis by constructing the Hesse matrix, which for this system is a symmetric $24 \times 24$ matrix. Since the energy is invariant under rotations about the $z$-axis, one eigenvalue must be zero. We call a state satisfying (2) "stable" if the Hesse matrix has only positive eigenvalues apart from one zero eigenvalue. This is equivalent to the fact that the given state provides a local minimum of the energy.

By applying this procedure to the two families of possible ground states of the icosahedron we determine numerically the above-mentioned stability ranges of the families: The 4- $\theta$-family is stable for $0 \leq M \leq 5.61441$ and the decagon family is stable for $0.54102 \leq M \leq 12$.

This implies that the system at $T \simeq 0$ will not immediately jump from the 4 - $\theta$-family into the decagon family if $B$ increases beyond $B_{\mathrm{c}}$ but remain in its family until $M>5.61441$. Conversely, the decagon family will remain the de facto spin configuration of the icosahedron if $B$ is lowered beyond $B_{\mathrm{c}}$ until $M<0.54102$. In fact, these hysteresis effects are observed in our simulational studies, see Fig. 1

Quantum calculations - We now discuss how the metamagnetic phase transition is manifested in the quantum Heisenberg icosahedron. Classically, the phase transition consists of a discontinuity of the magnetization as a function of the magnetic field. Quantum mechanically, the magnetization curve for $T=0$ is already a staircase of successive steps of unit height $\Delta M=1$, which result from crossings of levels with adjacent total magnetic quantum numbers $M$ and $M+1$. In the context of this phase transition we are looking for a magnetization jump of unusual height, i.e. $\Delta M>1$. It is clear that such a jump must occur because it occurs in the classical limit $s \rightarrow \infty$. The remaining questions therefore are, for which intrinsic spin quantum number $s$ is such a jump clearly visible, and are there other signs of the phase transition at smaller $s$ ?

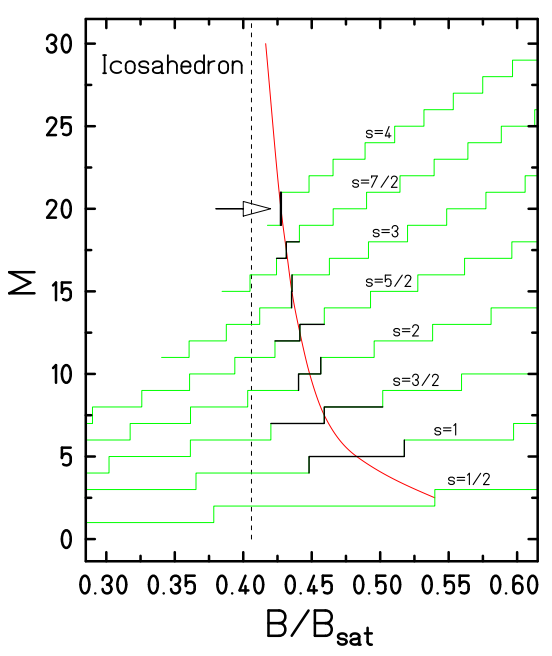

FIG. 3: Magnetization curves for $T=0$ and various values of the intrinsic spin quantum number $s$ : The magnetization plateaus of smallest width are highlighted on each curve. At $s=4$ a magnetization jump of $\Delta M=2$ occurs, marked by the arrow. The solid curve shows that the field values that bisect the smallest plateaus converge to the classical transition field (dashed line). For large $s$ the magnetization curve could only be evaluated down to a certain field due to prohibitively large dimensions of the related Hilbert spaces.

Using a Lanczos procedure which yields numerically exact lowest energy eigenvalues in subspaces with constant total magnetic quantum number $M$ we are able to evaluate magnetization curves at $T=0$ for various intrinsic spin quantum numbers. Figure 3 shows the relevant parts of the magnetization curves at $T=0$ for $s=1 / 2, \ldots, 4$. For integer values of $s$ the magnetization plateau of smallest width is highlighted; for half-integer values of $s$ we highlight two such plateaus. With increasing $s$ these widths shrink and already at $s=4$ a magnetization jump of $\Delta M=2$ occurs. This corresponds to a non-convex part of the discrete energy levels versus $M$.

In Fig. 3] we also provide a curve which bisects the magnetization plateaus of smallest width. Assuming that the bisector value of $B / B_{\text {sat }}$ is described by a polynomial in $1 / s$, we obtain as an estimate for the classic transition field $B / B_{\mathrm{c}} \approx 0.40 \pm 0.01$, which is in very good agreement with the classical result (see Table I). The uncertainty originates from the limited number of data points (eight) as well as from fluctuations between integer and halfinteger values of $s$.

Classical finite-temperature simulations - We have restricted our investigation to the determination of the lifetime of the high-field phase (decagon-family) in its 
metastable regime $\left(B / B_{\mathrm{c}}<1\right)$. The lifetime has been determined by the following procedure: First, the system is prepared in the decagon phase at $T=0$. Then the field is lowered to a value below the critical field value

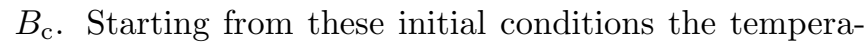
ture is set to a value $T>0$ and the trajectory of the system is calculated numerically by solving the stochastic Landau-Lifshitz equation.

Shown in the inset of Fig. 4 is a single trajectory of a sample system. We exploit the unique property of the decagon family that two spins persist in pointing in the direction of $\vec{B}\left(s_{z}(1) \approx s_{z}(2) \approx 1\right)$ until abruptly breaking away. This allows one to obtain an accurate determination of the decay time for this system. By performing $10^{5}$ such runs for each choice of $T$ and analyzing the histograms of the resulting decay times one can determine the lifetime distribution. A common measure is the socalled survival probability $P_{\mathrm{s}}(t)$ which is the probability of the metastable state not having decayed by the time $t$. In Fig. 4 we have plotted $P_{\mathrm{s}}(t)$ for the metastable state for various temperatures and an external field in the metastable regime. An appropriate choice for the lifetime, $t_{s}$, is the root of $P_{s}\left(t_{s}\right)=0.5$. We find that $t_{s}$ increases with decreasing temperature and appears to diverge for $T \rightarrow 0$ as $1 / T$. Although one obtains similar probability distributions for systems showing thermally activated magnetization switching [10], we emphasize that our model Hamiltonian does not contain any additional energy term providing an energy barrier. In fact, it is the special geometry of the icosahedron that causes the system to show metastability.

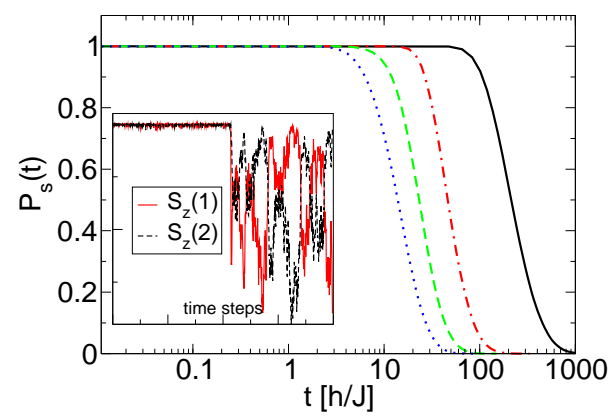

FIG. 4: Survival probability $P_{\mathrm{s}}(t)$ for the metastable decagon phase subject to an external field $B / B_{\text {sat }}=0.27$ for temperatures $k_{B} T / J=0.025,0.015,0.005,0.0005$ (left to right). Inset: Example trajectory of the system's time evolution at finite temperature.

Summary and outlook - In this Letter we have shown that the antiferromagnetic Heisenberg spin icosahedron undergoes a metamagnetic phase transition and displays rich hysteresis and metastability phenomena when sub- ject to a varying external field. Given this, the metamagnetic transition of the Heisenberg icosahedron may be of interest for potential applications in the area of nanomagnetic switches. It is also relevant in connection with magnetocalorics, since the magnetization jump is accompanied by an enhanced magnetocaloric effect [11, 12]. It is therefore very encouraging that recent progress in the synthesis of magnetic molecules offers the prospect of realizing the Heisenberg icosahedron [13, 14].

Acknowledgment - We thank Peter Hage for the use of his Lanczos diagonalization routine and R.E.P. Winpenny for discussions on the possible synthesis of a Heisenberg icosahedron. Ames Laboratory is operated for the U.S. Department of Energy by Iowa State University under Contract No. W-7405-Eng-82.

* Electronic address: christian.schroeder@fh-bielefeld.de

[1] J. Greedan, J. Mater. Chem. 11, 37 (2001).

[2] U. Schollwöck, J. Richter, D. Farnell, and R. Bishop, eds., Quantum Magnetism, vol. 645 of Lecture Notes in Physics (Springer, Berlin, Heidelberg, 2004).

[3] Y. Narumi, K. Katsumata, Z. Honda, J.-C. Domenge, P. Sindzingre, C. Lhuillier, Y. Shimaoka, T. C. Kobayashi, and K. Kindo, Europhys. Lett. 65, 705 (2004).

[4] J. Schulenburg, A. Honecker, J. Schnack, J. Richter, and H.-J. Schmidt, Phys. Rev. Lett. 88, 167207 (2002).

[5] C. Schröder, H. Nojiri, J. Schnack, P. Hage, M. Luban, and P. Kögerler, Phys. Rev. Lett. 94, 017205 (2005).

[6] C. Lhuillier and G. Misguich, High Magnetic Fields (Springer, Berlin, 2002), pp. 161-190.

[7] V. P. Antropov, S. V. Tretyakov, and B. N. Harmon, J. Appl. Phys. 81, 3961 (1997).

[8] H.-J. Schmidt and M. Luban, J. Phys. A: Math. Gen. 36, 6351 (2003).

[9] H.-J. Schmidt, J. Schnack, C. Schröder, and M. Luban, unpublished.

[10] G. Brown, M. A. Novotny, and P. A. Rikvold, J. Appl. Phys. 87, 4792 (2000).

[11] O. Derzhko and J. Richter, Phys. Rev. B 70, 104415 (2004).

[12] M. E. Zhitomirsky and A. Honecker, J. Stat. Mech.: Theor. Exp. p. P07012 (2004).

[13] A. J. Blake, R. O. Gould, C. M. Grant, P. E. Y. Milne, S. Parsons, and R. E. P. Winpenny, J. Chem. Soc.-Dalton Trans. pp. 485-495 (1997).

[14] E. K. Brechin, A. Graham, S. G. Harris, S. Parsons, and R. E. P. Winpenny, J. Chem. Soc.-Dalton Trans. pp. 3405-3406 (1997).

[15] We invite the reader to examine movies of our simulations at http://www.fh-bielefeld.de/fb2/schroeder

[16] This is confirmed by numerical methods [9]. 